\title{
An ARM-based debugging system for multi- pole array acoustic logging tools
}

\author{
Lu Junqiang ${ }^{1,2}$, Ju Xiaodong ${ }^{1,2 *}$ and Men Baiyong ${ }^{1,2}$ \\ ${ }^{1}$ State Key Laboratory of Petroleum Resources and Prospecting, China University of Petroleum, Beijing 102249, China \\ ${ }^{2}$ Beijing Key Laboratory of Earth Prospecting and Information Technology, China University of Petroleum, Beijing 102249, \\ China
}

(C) China University of Petroleum (Beijing) and Springer-Verlag Berlin Heidelberg 2014

\begin{abstract}
This paper presents a debugging system for multi-pole array acoustic logging (MPAL) tools. The debugging system proposed in this study can debug the MPAL tool system, sub-system and local electronics. In the test equipment, we have used principal and subordinate structures, and interconnected the host computer and the front-end machine via Ethernet. The front-end machine is based on the ARM7 (advanced reduced instruction set computing (RISC) machine) technique, the processor of which runs an embedded operating system, namely, uClinux OS. We have analyzed the system telecommunication, human-machine interface circuit, transmitter mandrel interface circuit, receiver mandrel interface circuit, and board-level test interface circuit. The software used in the system consists of the embedded front-computer software and the host application software. We have explained in detail the flow chart of the boot loader in the embedded front-computer software. The host application software is composed of four application subroutines, which match with the functional modules of the system hardware. A net communication program based on the server/client mode is implemented by means of socket programming and multi-thread programming. Test results indicate that the data transmission rate of the system is higher than $1 \mathrm{MB} / \mathrm{s}$, which completely meets the current requirements of the data transmission rate between the tool system and the wireline telemetry device. Application of the debugging system, which includes multiple level test methods, shows that the proposed system can fully meet the test requirements of MPAL at various levels.
\end{abstract}

Key words: Acoustic logging tool, debug system, advanced RISC machines, embedded technique, network interconnection

\section{Introduction}

Multi-pole array acoustic logging (MPAL) is one of the most advanced acoustic logging technique that has been successfully developed and applied to the field of oil and gas exploration and production (Chen and Tang, 2012; Ju et al, 2008; Lu, 2007; Lu et al, 2008; Qiao et al, 2011; Su et al, 2011; Tang et al, 2007; Tang and Cheng, 2004; Tang and Patterson, 2009; Tang and Wei, 2012; Wang et al, 2012; 2013). In the production of MPAL tools, verification and calibration are important processes that ensure the feasibility and overall performance of the tool. From the viewpoints of structure and associated electronics, MPAL is more complex than other acoustic tools, and includes many functional modules, such as the control electronics, receiver mandrel, sound isolation, transmitter mandrel, transmitter electronics, and so on. The control electronics consist of system interface circuits, controlling circuits, acquisition circuits, analog signal

*Corresponding author. email: juxdong@cup.edu.cn

Received December 3, 2013 receiving and processing circuits, and local communication circuits (Lu, 2007; Lu et al, 2008). In order to ensure efficient manufacturing of high quality tools and subsequent field use, it is highly important to study the test methods that include multiple levels of tool system, sub-system, and local components. The test methods have to be targeted for diagnosis and calibration of the tools (Ju et al, 2009).

A debugging system has become an important part of communication, electronics and other fields (Chen et al, 2013; Ye et al, 2013). During the last few years, there has been a significant increase in the complexity of the electronics systems and detectors of logging tools. This has obviously increased the demand for a professional testing system. Although the international geophysical service corporations provide special on-site debugging systems for logging tools, details of the functioning of the system have not been disclosed in the literature. Ju and his co-workers $(\mathrm{Ju}$ and $\mathrm{Li}$, 2005; Ju et al, 2009; 2010) conducted extensive studies of debugging systems for logging tools and proposed a system architecture based on an embedded system. The embedded system runs on the embedded $\mu \mathrm{C} / \mathrm{OS}$-II real-time operating 
system. They designed an extended structure with reference to the PC104 industrial plug. Test interface circuits for pads of a micro-resistivity logging tool were achieved with these technologies. In the present study, we have proposed and implemented for the first time an ARM-based debugging system for MPAL, which can realize the three test levels. These are the tool system, sub-system, and board level. The proposed debugging system is interconnected with the host PC through a network, with the system bus or a serial bus connecting every part of the system. Besides, the debugging system proposed in this study offers the advantages of easy maintenance and feasibility to upgrade and extend. Besides the high voltage circuit can be isolated from the low voltage circuits, thereby offering better electromagnetic compatibility. The proposed debugging system can overcome the shortcomings associated with traditional computer board, which include the difficulties in hot swapping, extending, and short distance interconnection.

In this paper, the tool system debug tests the system communication, while the sub-system debug tests the main controlling electronics, receiver mandrel and transmitter mandrel, and the local components debug mainly aims at the system control circuits, analog signal receiving and acquisition circuits. All the objects in the three test levels are complex parts of the tool system, which decide the tool performance.

\section{Basic principles of the proposed debugging system}

The main function of the transmitter mandrel is to excite suitable acoustic signals, which can be evaluated by testing the sound pressure, basic frequency, and directivity of the transducers. Here, the basic frequency can be measured using an impedance analyzer, while the sound pressure and directivity can be obtained from an acoustic tank. The free field voltage sensitivity level of the hydrophone is as follows:

$$
M_{\mathrm{el}}=20 \lg \frac{M_{\mathrm{e}}}{M_{\mathrm{e} 0}}(\mathrm{~dB})
$$

where $M_{\mathrm{e}}$ is the free field voltage sensitivity measured in $\mathrm{V} / \mathrm{Pa} ; M_{\mathrm{e} 0}$ is the reference value of the voltage sensitivity, which is measured with the same unit as that of $M_{\mathrm{e}}$. In general, $M_{\mathrm{e} 0}=1 \mathrm{~V} / \mu \mathrm{Pa}=10^{6} \mathrm{~V} / \mathrm{Pa}$. Accordingly, the free field voltage sensitivity level becomes

$$
M_{\mathrm{el}}=20 \lg M_{\mathrm{e}}-120(\mathrm{~dB})
$$

The free field sound pressure of the plane wave is

$$
p_{\mathrm{f}}=\frac{e_{\mathrm{oc}}}{M_{\mathrm{e}}}=\frac{e_{\mathrm{oc}}}{10^{\left(M_{\mathrm{el}}+120\right) / 20}}
$$

where $e_{\mathrm{oc}}$ is the open circuit voltage of the hydrophone measured in $\mathrm{V}$.

In principle, the consistency of the receiving array transducers is very important for the receiving mandrel, which can be evaluated by testing the sensitivity, frequency, and directivity. The frequency can be measured using an impedance analyzer, while the sensitivity and directivity can be obtained in the acoustic tank.

The sensitivity of the receiving piezoelectric vibrators is often measured using the comparison method. First a standard hydrophone is placed in the radiation field of the transmitting transducer, and its open circuit voltage $\left(e_{\mathrm{oc}}\right)_{\mathrm{H}}$ is measured. Subsequently, the receiving piezoelectric vibrator is placed in the same location of the sound field, and its open circuit voltage $\left(e_{\mathrm{oc}}\right)_{\mathrm{X}}$ is measured under the same test conditions. Accordingly, the free field voltage sensitivity of the hydrophone $\left(M_{\mathrm{e}}\right)_{\mathrm{H}}$ and that of the receiving piezoelectric vibrator $\left(M_{\mathrm{e}}\right)_{\mathrm{X}}$ are respectively

$$
\begin{aligned}
& \left(M_{\mathrm{e}}\right)_{\mathrm{H}}=\frac{\left(e_{\mathrm{oc}}\right)_{\mathrm{H}}}{p} \\
& \left(M_{\mathrm{e}}\right)_{\mathrm{X}}=\frac{\left(e_{\mathrm{oc}}\right)_{\mathrm{X}}}{p}
\end{aligned}
$$

From the above-mentioned expressions, $\left(M_{\mathrm{e}}\right)_{\mathrm{X}}$ can be expressed as

$$
\left(M_{\mathrm{e}}\right)_{\mathrm{X}}=\frac{\left(e_{\mathrm{oc}}\right)_{\mathrm{X}}}{\left(e_{\mathrm{oc}}\right)_{\mathrm{H}}}\left(M_{\mathrm{e}}\right)_{\mathrm{H}}
$$

Implementing logarithmic calculation to the above expression, and considering the reference value of the voltage sensitivity in water as $M_{\mathrm{e} 0}=1 \mathrm{~V} / \mu \mathrm{Pa}$, the free field voltage sensitivity level of receiving piezoelectric vibrators is given as

$$
\left(M_{\mathrm{el}}\right)_{\mathrm{X}}=20 \log \frac{\left(M_{\mathrm{e}}\right)_{\mathrm{X}}}{\left(M_{\mathrm{e}}\right)_{0}}=20 \log \frac{\left(e_{\mathrm{oc}}\right)_{\mathrm{X}}}{\left(e_{\mathrm{oc}}\right)_{\mathrm{H}}}+\left(M_{\mathrm{el}}\right)_{\mathrm{H}}
$$

where $\left(M_{\mathrm{el}}\right)_{\mathrm{H}}$ is the free field voltage sensitivity level of the standard hydrophone expressed in $\mathrm{dB}$.

MPAL requires high consistency of the analog channels, and hence demands the testing of eight analog signal receiving and processing channels. A phase-sensitive demodulation algorithm is applied to extract signal amplitude and phase of each channel (Men et al, 2011), and the consistency of all the channels can be estimated.

The input signal can be expressed as

$$
d(t)=A \cos \left(\omega_{i} t-\varphi\right)+d
$$

Assuming that the time sampling length is $T$, the Fourier transform of the above expression is (Wang, 2012)

$$
F(\omega)=\int_{0}^{T} d(t) \mathrm{e}^{-j \omega t} \mathrm{~d} t=\int_{0}^{T} d(t) \cos \omega t \mathrm{~d} t-j \int_{0}^{T} d(t) \sin \omega t \mathrm{~d} t
$$

If $\omega=\omega_{i}$, then the real part $R$ and the imaginary part $X$ in the frequency point $\omega_{i}$ can be calculated as

$$
\begin{aligned}
& R=\int_{0}^{T} d(t) \cos \omega_{i} t \mathrm{~d} t=\frac{T}{2} A \cos (\varphi) \\
& X=\int_{0}^{T} d(t) \sin \omega_{i} t \mathrm{~d} t=\frac{T}{2} A \sin (\varphi)
\end{aligned}
$$

If the sampling point is $N$, then the discretization expressions are 


$$
\begin{aligned}
& R=\sum_{n=1}^{N} d(\Delta T n) \cos \left(\omega_{i} \Delta T n\right) \Delta T=\frac{\Delta T N}{2} A \cos (\varphi) \\
& X=\sum_{n=1}^{N} d(\Delta T n) \sin \left(\omega_{i} \Delta T n\right) \Delta T=\frac{\Delta T N}{2} A \sin (\varphi)
\end{aligned}
$$

Accordingly, we can get the following expressions

$$
\begin{aligned}
& A \cos (\varphi)=\frac{2}{N} \sum_{n=1}^{N} d(n) \cos \left(\omega_{i} \Delta T n\right) \\
& A \sin (\varphi)=\frac{2}{N} \sum_{n=1}^{N} d(n) \sin \left(\omega_{i} \Delta T n\right)
\end{aligned}
$$

where $d(n)$ is the sampling point $n$.

Using this, the amplitude $A$ and phase $\varphi$ can be calculated, based on which the consistency of all the channels can be analyzed.

\section{Overall structure of the proposed debugging system}

Based on every step of the tool manufacturing process and field application, the proposed debugging system can support several levels of debugging and testing, such as the tool system level, sub-system level, and local components level (Ju et al, 2009; 2010; Ju and Li, 2005; Lu et al, 2012). According to the protocol of the system interconnection interface, the system-level debugging module downloads control commands to the tool and gets data from the tool. The received data are processed to determine the operating status of the tool. Besides, all the functions of the field control software are also realized in the system-level debugging module. On the other hand, the subsystem-level debugging module mostly aims at transmitter electronics, transmitter mandrel, receiver mandrel, and measurement and control electronics. The local circuit-level debugging function supports the debugging of receiving circuit, acquisition circuit, and DSP (digital signal processor) measurement and control circuit.

Considering the technical characteristics of modern electronic systems and to take the most advantage of hardware and software sources of the present mainstream PC (personal computer), we have designed a system architecture based on ARM and IEEE 802.3 (Ethernet) (Gong et al, 2012; Jin et al, 2010). Taking into account the functional requirements of the front-end subsystem, it is undoubtedly a good proposal to use the master-slave mode, also called as the host computer and front-end machine mode system architecture in the testing system. This proposal is based on the linking of building block organization and is very conducive for further expansion of the function. A high performance notebook PC with an X86 family processor is used as the host computer. The host computer, front-end machine, and other external equipment, constitute the multiple joint virtual peer-to-peer network, forming a star connection. The overall block diagram of the proposed debugging system is shown in Fig. 1.

As shown in the figure, the main functional module includes notebook PC, ARM7 main control board, tool system communication controller, local man-machine interface, transmitter mandrel interface, receiver mandrel interface, board-level test interface, hot-swap control interface, and special power supply for the downhole tool. The tool system interface is used to debug the entire tool, and it provides tool power supply and high speed CAN (controller area network) interface. Besides, the DTB (data transfer bus) communication interface is also designed to ensure compatibility with the CNPC (China National Petroleum Corporation) EILog05 system.

The core of the main controller board is the ARM7TDMI S3C44B0X chip manufactured by SAMSUNG Corporation (Samsung Electronics, 2001), which also includes program storage, data storage, file system storage, Ethernet interface, UART (universal asynchronous receiver/transmitter) interface, encoder and extended bus interface. The link between the host computer and the main controller board is realized using a $10 \mathrm{M}$ Ethernet network. The functional control of the circuit board and bidirectional data exchange are realized by means of a high-speed extended bus, which consists of address bus, data bus, control signals, bus interface signals, system clock, DMA (direct memory access), and interrupt signals. The extended bus connects the expansion board using high-speed buffering drivers, which can guarantee reliable signal transmission. During real time operation, the host can send control commands to the front-end machine, which in turn can rapidly upload data to the host at the same time. Related human-computer interaction and subsequent processing tasks are accomplished using a high-performance PC. The two UART interfaces of the main control board are respectively used for the hyperterminal interface and power control of the downhole tool. The hyperterminal not only can watch the running state of the system, but can also send data and other working parameters to the system.

\section{Hardware design of the functional circuit board}

The functional circuit boards are interconnected through the high speed extended bus. The linking structure looks like building blocks, and the corresponding test can be achieved. The functional circuit boards mainly consist of system communication, human-computer interface, receiver mandrel test interface, transmitter mandrel test interface, and board-level test interface.

\subsection{System communication and man-machine interface circuit}

System communication and man-machine interface are important functions of the functional circuit board that are used to test the tool system, the structural diagrams of which are shown in Fig. 2. As is seen, the Altera cyclone series EP1C12Q240 (200,000 logic gates) field programmable gate array is used as the core control component, which implements the CAN interface, DTB interface, LCD (liquid crystal display) interface, and the keyboard interface functions. This board contains two CAN channels and one DTB bus interface, all of which could be used as main controllers (master mode) as well as in tool slave mode, facilitating the assembly of several boards. For instance, this board could substitute for the CAN or DTB telemetry 


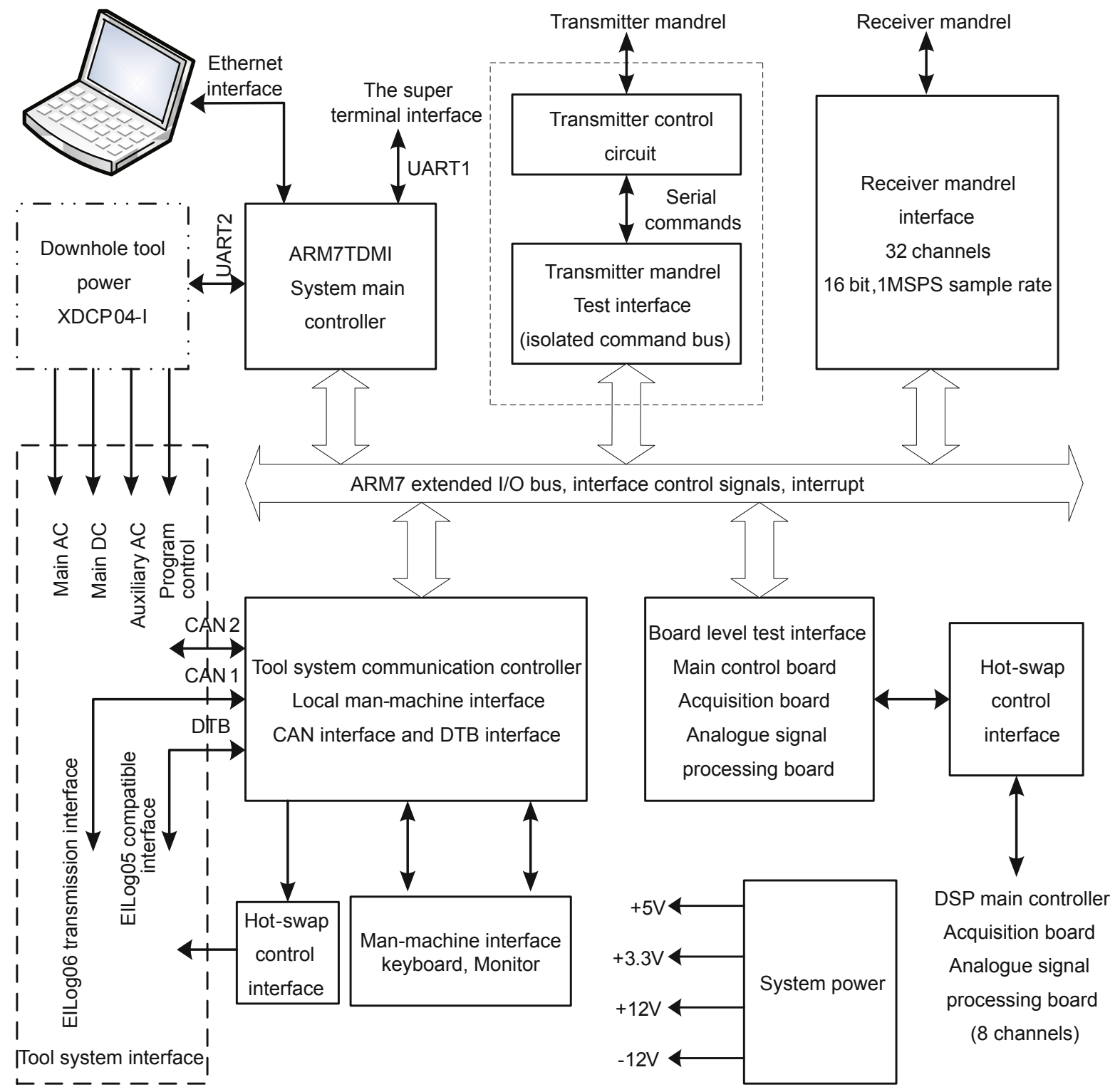

Fig. 1 Overall structural diagram of the proposed debugging system

modems (Liu et al, 2011; Yang and Cai, 2011) in detecting and checking the whole downhole tools, as well as simulating multiple downhole tools to provide debugging data for the actual telemetry modems. Complete photoelectric isolation is implemented in the circuit design, which has higher security and reliability. In this study, the one channel CAN bus interface works as the main control mode regulating the whole tool system.

The board also contains a standard LCD interface and a special keyboard interface, which could be used for local human-computer interaction. The LCD and keyboard serve to assist the tool operation and control the test system. The command operation could be achieved using the upper computer or the local keyboard and the LCD interface. In most cases, the front-end system runs in black box mode, so that the human-computer interaction is achieved using the upper computer, while the LCD displays the state of the test system.

\subsection{Transmitter mandrel interface circuit}

Fig. 3 shows the schematic diagram of the transmitter mandrel interface circuit ( $\mathrm{Lu}$ et al, 2010). It is mainly composed of the data bus buffer, clock, CPLD (complex programmable logic device) (Altera, 2009; Lattice, 2006), optical isolation, and differential driver. The CPLD acts as an interface between the transmitter interface circuit board and ARM core circuit board, which receives and decodes the control commands received from the ARM extended bus. Following that, the output of the CPLD provides control commands in sequence according to the pre-defined serial command protocol. The control commands can set the working parameters, such as working mode, transmitter mode selection, pulse width, repetitive work cycle, and firing interval. When the tool control mode is set, the control command bus of the tool system automatically sets all the working parameters. 


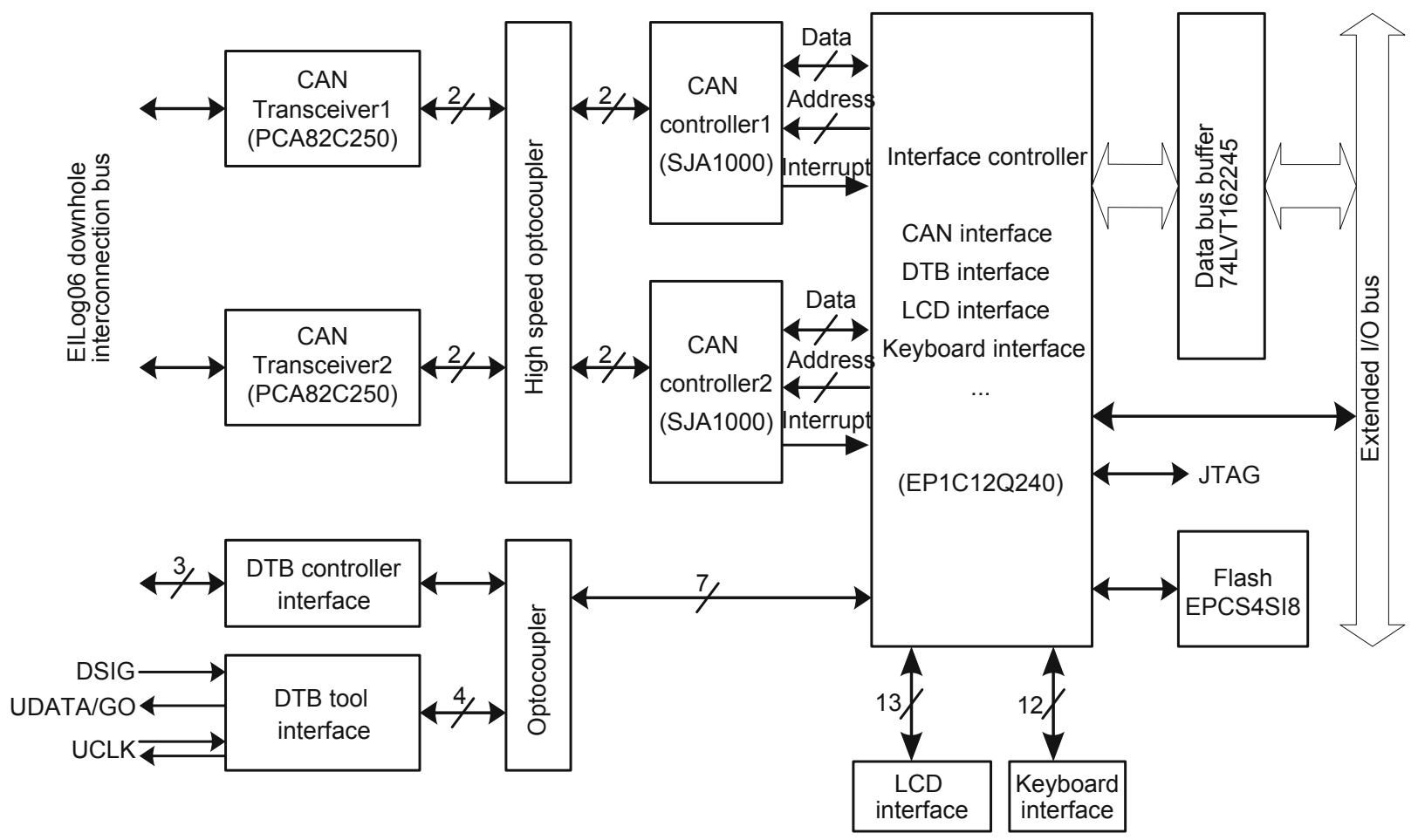

Fig. 2 Structural diagram of the system communication and man-machine interface

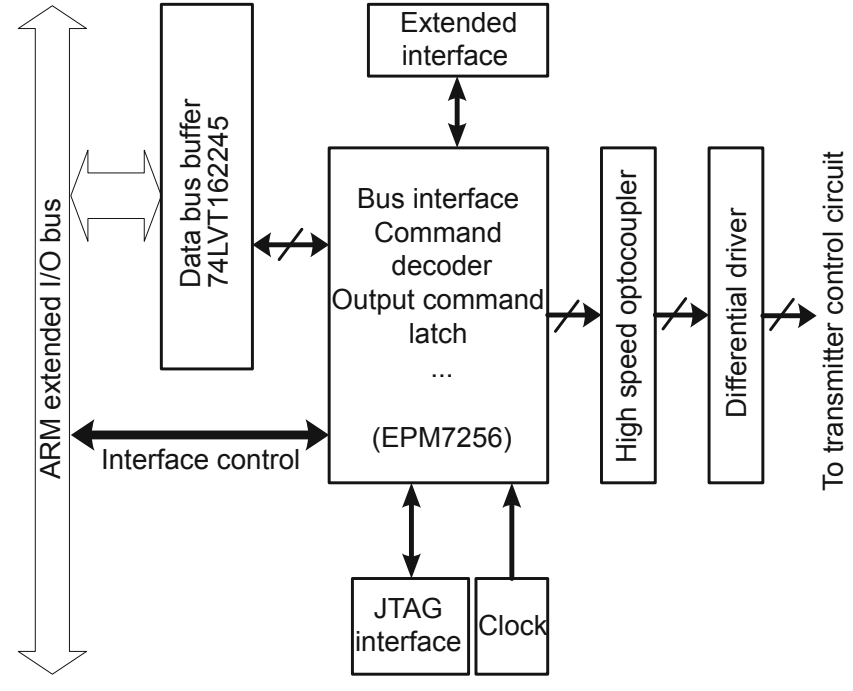

Fig. 3 Schematic diagram of the transmitter mandrel interface

The output control signals of the transmitter interface circuit are connected with the main control system through the high-speed optocoupler for electrical isolation. The optocoupler serves to effectively reduce the interference between each functional circuit and at the same time protect the ARM main control system from the high voltage generated by the transducer excitation. The isolated output signals thus obtained are in the form of differential transmission. The transmission mode has the characteristics of good anti-interference ability, reliable transmission, and high speed, achieving point-to-point (driving mode) and point-to-multipoint (receiving mode) signal transmission mode.

\subsection{Receiver mandrel interface circuit}

Fig. 4 shows the schematic diagram of the receiver mandrel interface circuit. The receiver mandrel of the MPAL includes eight sets of transducers, with every set consisting of four transducers. In each set two transducers are in $X$ orientation $(X+, X$-), while the remaining two are in $Y$ orientation $(Y+, Y-)$. Therefore, a total of 32 transducers need to be tested to achieve performance and consistency (Che et al, 2005; Qiao et al, 2006; Wu et al, 2012; 2013). The CPLD (EPM1270) realizes the bus interface, sends internal commands, and controls the acquisition and channel switching. The working parameters and timing sequences of the tool can be set through the internal control command bus that runs through the whole tool. The master control system sends control commands to the CPLD via the ARM expansion bus. Once the startup command is received, the CPLD, in turn, generates serial commands in the format required by MPAL. The isolation amplifier AD215, which features a $-3 \mathrm{~dB}$ full-power bandwidth of $120 \mathrm{kHz}$, provides complete galvanic isolation between the analog signal processing channel and the analog-to-digital converter. Meanwhile, the digital control signals from the CPLD, such as the acquisition control signals and channel switch signals, are isolated photoelectrically. All these isolation measures reduce the interference (Liu et al, 2013; Peng et al, 2011), and guarantee precision. Besides, the photoelectric isolation designed for the internal control command from CPLD not only reduces the interference, but also establishes safe connection between the MPAL tool system and the test control system, thereby avoiding any undesirable effects caused due to break down. 


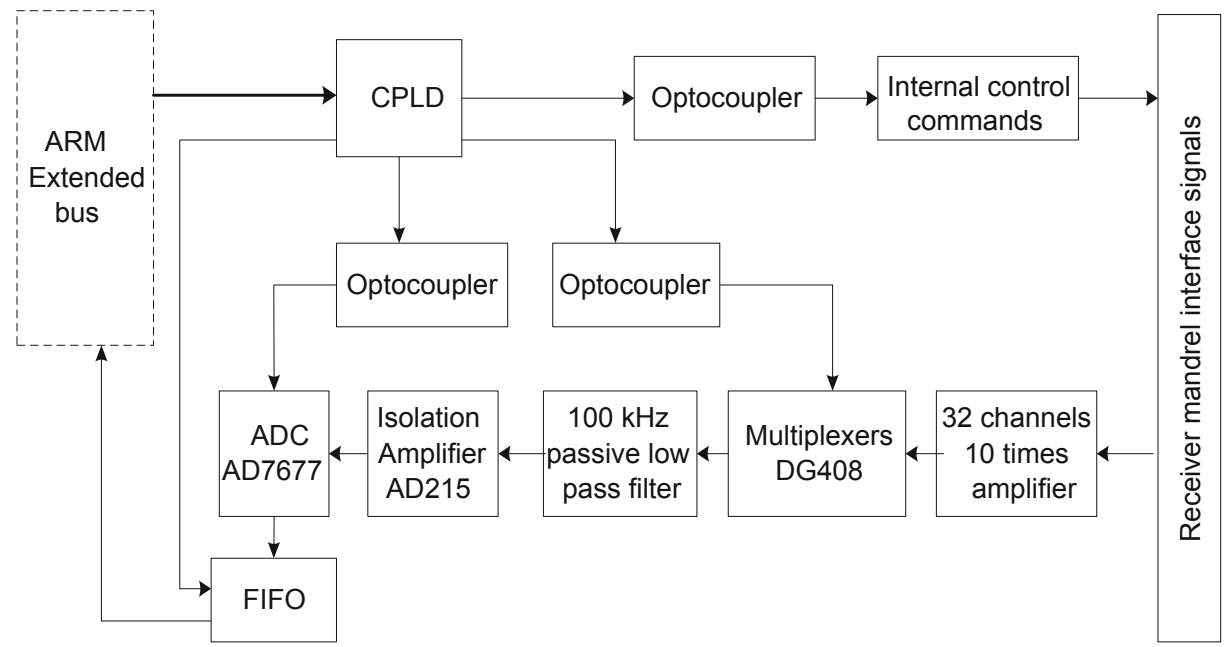

Fig. 4 Schematic diagram of the receiver mandrel interface circuit

\subsection{Board level interface circuit}

The board level test is mainly for the system control circuit, and the analog signal receiving and processing circuit, which are the most important circuits of MPAL featuring high integration and complexity. These two circuits determine the entire control performance of the tool, the dynamic range, consistency of several analog channels, and the precision of the system. Fig. 5 shows the principle of the interface circuit used for board level testing. All the test signals support hot plugging, to allow the easy operation and protection of the test system and the circuits under test.

The 16-channel acoustic signals and the serial commands set to channels serve as the inputs of the analog signal receiving and processing circuit, while the digitized data are the outputs. The performance of each channel can be assessed via changing the frequencies of the analog waves from the signal source using the signal selection switches. The filtered signals can be attenuated by $1,1 / 10$, or $1 / 100$ for the test of amplification at different levels. Furthermore, the output signals can often be a combination of the same phase or the opposite phase. This combination is used to simulate the monopole, dipole, or quadrupole acoustic signals. The serial command bus and the data transfer bus are managed by the CPLD, based on the parameters obtained from the

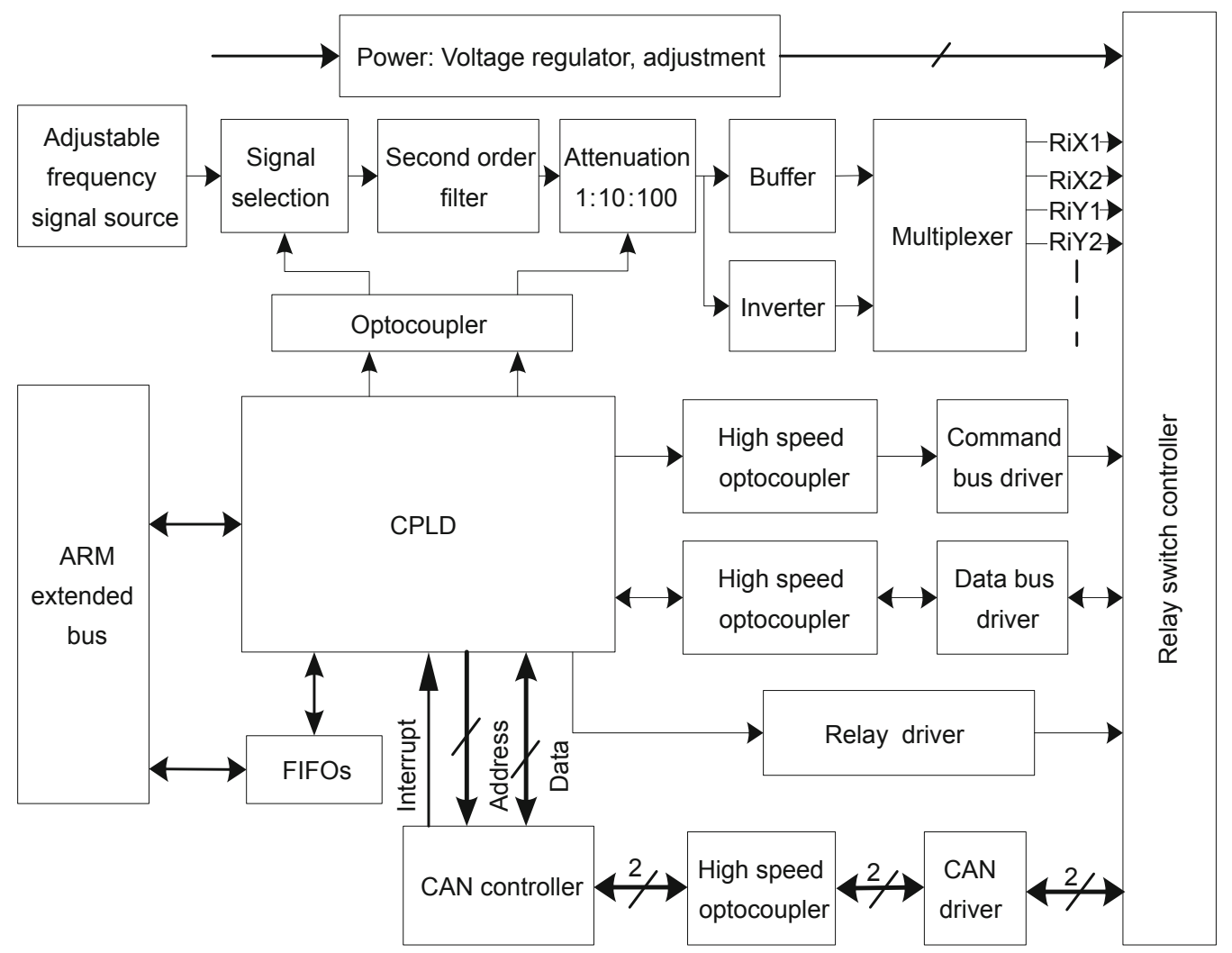

Fig. 5 Schematic diagram of the board level test 
ARM. Subsequently, the buses download the channel setting parameters and acquisition parameters in serial mode, read the acquired data, store them into the FIFO (first in first out) memory after the acquisition cycle, and then upload the data in FIFO via the extended ARM bus.

The input interface signal of the system control circuit is a high-speed serial data bus from which the main controller reads the data, while the output interface is the CAN bus of the tool system. The high-speed serial data bus is imitated by the CPLD, which generates simulation data in the form required by the acquisition circuit. Both the wave data downloaded by the host computer and the special simulation data from the front-end machine can act as the acquired data. The CAN bus interface imitates the function of the downhole telemetry circuit and runs in accordance with the formats of the downloaded commands or the uploaded data of the downhole tool system. The downloaded commands and simulation data obtained from the high-speed serial data bus are uploaded through the CAN bus. Subsequently, the host computer or the front-end machine estimate the operating status of the system control circuit based on the received data. On the other hand, the front-end machine can also download special test commands to the master control board via CAN bus to independently examine some local circuit, such as RAM (random access memory) or FIFO memory.

\section{Software design}

The software includes computer (host) application software and embedded front-computer software.

\subsection{Embedded front-computer software}

The embedded operating system (EOS) forms the core of the embedded front-computer software, which completes the real-time multi-task scheduling, data processing, and reliable communication between tasks in the particular user environment. The embedded front-computer software consists of real-time operating system (RTOS) kernel transplantation, boot loader (BL) program, special hardware drivers (such as the character device driver), TCP/IP (transmission control protocol/internet protocol) network protocol stack transplantation, and user application program design.

In this study, we used the EOS uClinux, which possesses most properties of Linux (Matthew and Stones, 2007) and is widely used in embedded systems. The operating system can be used in the processor without a memory management unit (MMU). The most remarkable characteristic of the operating system is its small kernel, high efficiency, open source code, complete TCP/IP network protocol stack, and underlying drivers, supporting a variety of network interface chips. The Linux operating system includes common device drivers, such as character device drivers, block device drivers, and network device drivers. For general applications, the corresponding driver can be transferred to the specific hardware driver, without any requirement of writing it in the driver. The Linux network system is mainly based on the BSD (Berkeley software distribution) UNIX socket. The data transportation between the application and the driver can be completed by means of special data structures. The Linux system supports buffering of sent and received data, and provides streaming transmission mechanism. Since Linux includes the driver to the interface chip and the RTL8019 chip is used in the design, the driver can be directly transplanted and allocated according to the hardware resources. In the uClinux operating system, the network application software is developed on the basis of the popular BSD socket.

The boot loader (BL) program in the embedded frontcomputer software is similar to the BIOS (basic input output system) program of the PC motherboard. The BL program is stored in the NOR-Flash memory of the ARM7 main control board, and runs before the OS core and the application process. It essentially initializes the processor and the other circuits, and takes the hardware and the software into a suitable state. The flow chart of BL is presented in Fig. 6 . Firstly, the hardware should be initialized in the management model. The main initialization is to prohibit all interrupts, set the master system clock, configure the peripherals, and complete the multi-functional port configuration. After initialization, the next step is to configure the network connection, set the super terminal, map the memory space, set the special registers of CPU (such as PLL (phase locked loop) clock, watchdog timer, interrupt request, fast interrupt request), and enable the interrupts. The sequential operation of this protocol sets the hardware into a suitable state to download and run the operating system.

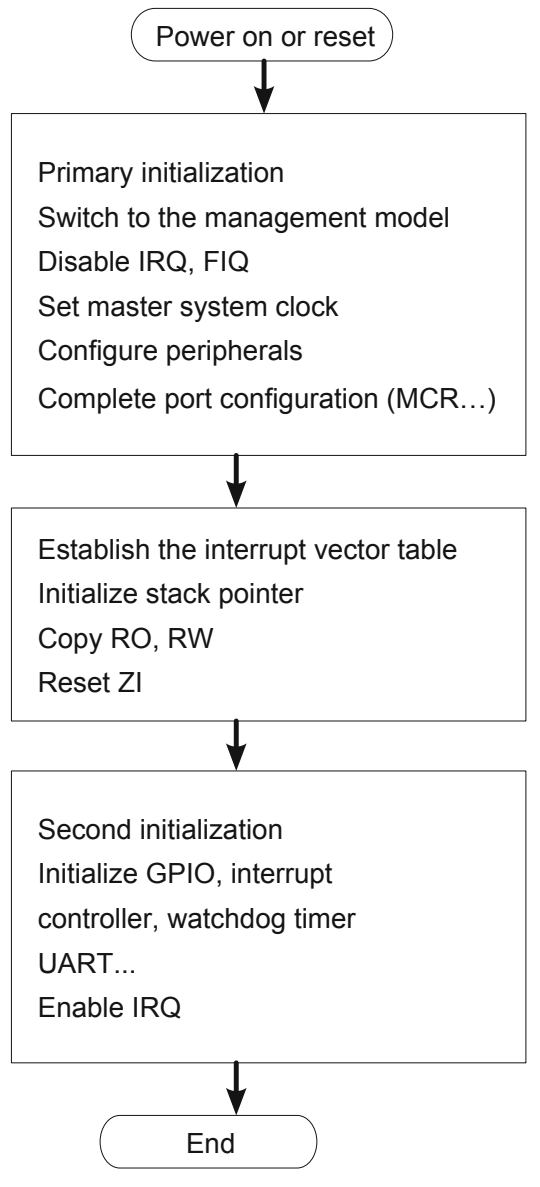

Fig. 6 Flow chart of BL 


\subsection{Host application software}

The development environments of host application software are Windows XP operating system and Visual Studio.Net, under which the object-oriented software is developed. Because the network connection does not need the actual hardware driver, all the codes run in the Ring3 layer of the X86 (non-privileged layer). Fig. 7 shows the structure of the host application software and its specific functional block diagram.

The host application software includes a total of four functional control units that manage the corresponding control circuits. The network communication management module is based on the TCP/IP protocol, which is realized with the use of the Winsock socket programming that can complete transmission of the application system commands, status, and test data. The socket interface consists of stream socket
(SOCK_STREAM), datagram socket (SOCK_DGRAM), and original socket. In the design, we used the stream socket and network connection mode of the server (PC)/client (frontend computer), which provide a reliable, connection-oriented communication flow to ensure the accuracy and orderliness of data transmission. The network communication management module is completed by the multi-threaded programming technique. The module can perform real-time detection of the network status. When there are data in the receiving buffer, the main process management module can get the data through the Windows message mechanism.

Test parameters are set by the human-machine interface in four control units. At the same time, the commands with fixed formats are generated according to the parameters. Data and commands are passed between the various control units and the main process module through the message loop. The

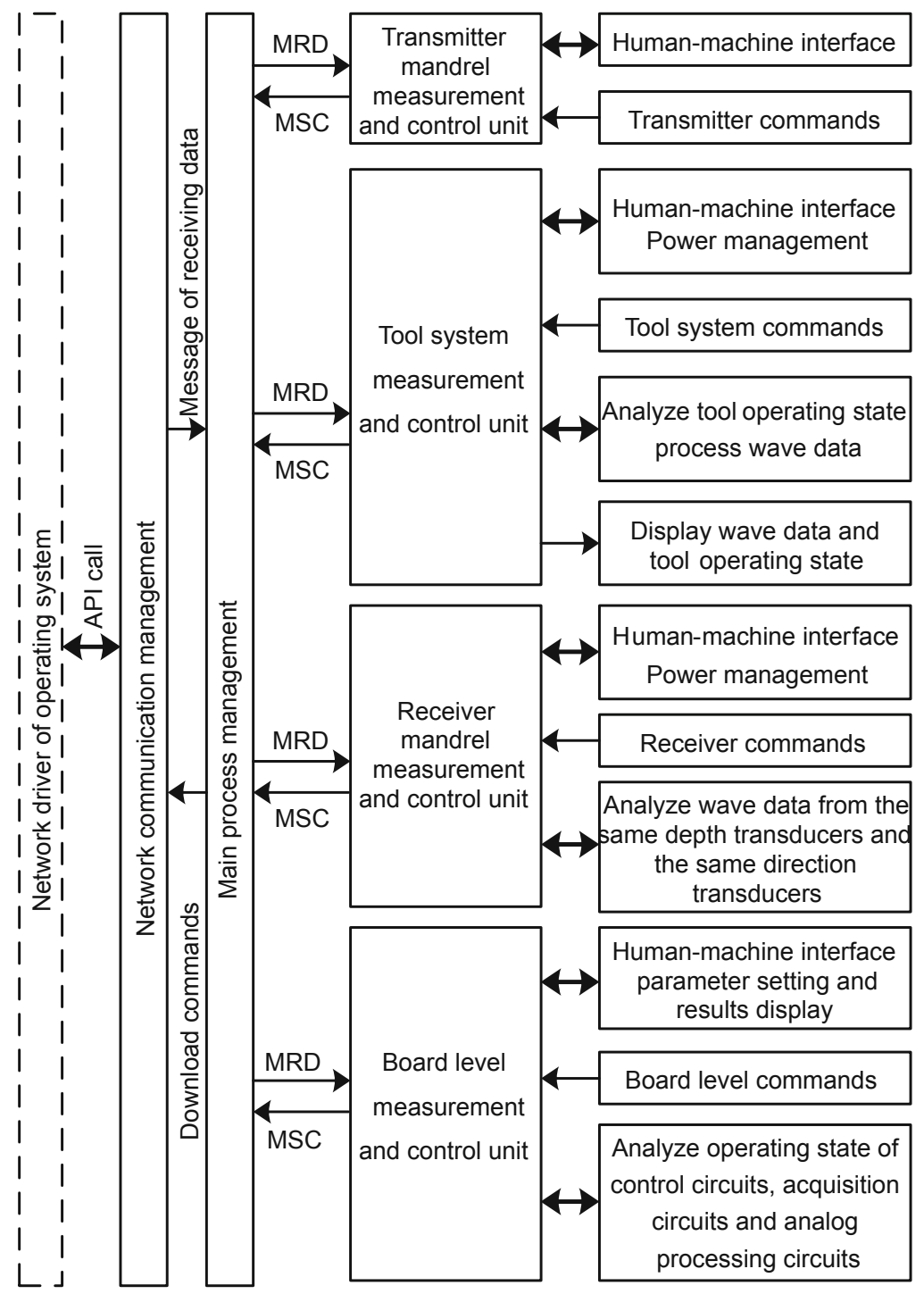

MSC: Message of sending commands

MRD : Message of receiving data

Fig. 7 Structure and specific functional block diagram of the host application software 
transmitter mandrel test control unit generates the selection commands of monopole, cross dipole, and quadrupole transmitting transducers, and generates the corresponding operating parameters of the transmitting transducer. The tool system test control unit realizes power management of the downhole tool, generates tool system commands, analyzes data, processes waveform data, and displays tool status and various models of the waveform. The human-machine interface and operating method of the unit are basically the same as that of the MPAL in the CNPC EIlog logging system, and the operator can easily use the on-site control system and the test system. The tool works in the same depth transducer test mode or the same direction transducer test mode according to the control commands obtained from the receiver mandrel test control unit. The circumferential transducer matching and characteristics are validated by analysis of the received data. In real time, approximately eight groups of transducers can be analyzed, with the possibility of analyzing eight transducers of the same direction (e.g., $X+$ direction) with different spacing. The board level test control unit is mainly used to test the main functional circuits of the MPAL. In a typical process, the parameters are downloaded according to the functional requirements of the unit circuits, the test data uploaded by the corresponding circuits are read, and then the operating states of the circuits are judged according to the received data.

\subsection{Network application software}

The design of network application between the upper computer and the embedded front-end computer is based on the network connection mode of the server (host computer) and the client (front-end computer). As shown in the procedure presented in Fig. 8, the client first creates a stream socket with the type of SOCK_STREAM, and then sets the IP address and connection port of the server. Following that, the client establishes a connection with the server via the API function connect. In response to this, the server specifies the version of the network sockets and retrieves details of the specific sockets implementation by WSAStartup function, creates a socket by socket function, binds the local IP address and connection port to the created socket, and then places a socket in a state in which it listens for an incoming connection using the listen function. The accept function permits an incoming connection attempt on a socket. It then creates and returns a handle to the new socket. After successfully establishing the connection between the client and the server, the recv function and send function can be used respectively to receive and send data. As the network data transmission may cause a blocking wait, we use multithreading programming techniques in the communication program of the embedded front-end computer and the host computer. The network communication is realized through the auxiliary thread, while the data processing and human-machine interaction are achieved through the main thread, so that the application can run efficiently and stably.

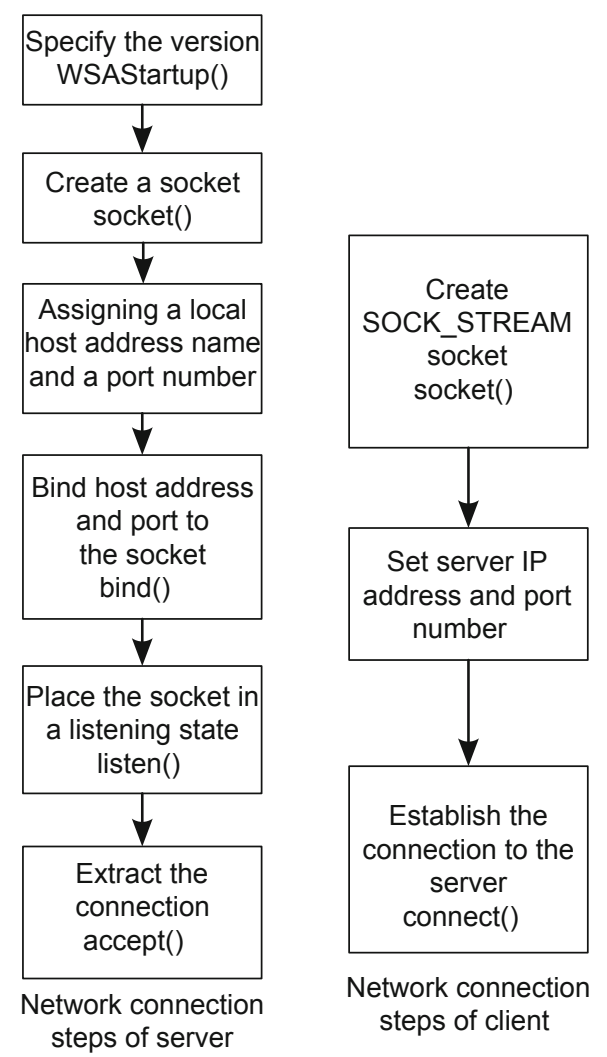

Fig. 8 Procedure for establishing the network connection of server/client

\section{Experimental results}

In order to verify the transmission bandwidth and response time, we tested the transmission rate of the application software, based on the network connection mode of the server and the client. After several tests, the average transmission performance is acquired. According to the results, the CPU utilization rate is $3 \%$ and the delay time for communication is $507 \mu \mathrm{s}$. When $1 \mathrm{~KB}$ data is transmitted, the receiving time of the client is $181 \mu \mathrm{s}$, the sending time of the client is $394 \mu \mathrm{s}$, the sending time of the server is $175 \mu \mathrm{s}$, and the receiving time of the server is $913 \mu \mathrm{s}$. Thus, if the sending and receiving rate is higher than $1 \mathrm{MB} / \mathrm{s}$ by either the client or the server, then the transmission rate achieves the transmission bandwidth of the network. The transmission bandwidth is significantly higher than that of the domestic mainstream cable telemetry system (less than $1 \mathrm{Mbit} / \mathrm{s}$ ), and hence can fully meet the requirements of the data transfer rate between MPAL and the telemetry system.

The transmitter mandrel of the MPAL comprises of monopole, dipole, and quadrupole transducers, the sound pressures and directivities of which can be tested to evaluate their performance. Experimental results of one transmitter mandrel are shown in Table 1. The sound pressures were tested before and after installation. Comparing the sound pressures before and after the installation, it can be realized that the structure and assembly of the transmitter mandrel are 
Table 1 Comparison of the sound pressures of one transmitter mandrel before and after installation

\begin{tabular}{cccc}
\hline & $\begin{array}{c}\text { Monopole } \\
\text { transducer }\end{array}$ & $\begin{array}{c}\text { Dipole } \\
\text { transducer- } X\end{array}$ & $\begin{array}{c}\text { Dipole } \\
\text { transducer- } Y\end{array}$ \\
\hline $\begin{array}{c}\text { Sound pressures } \\
\text { before installation, Pa }\end{array}$ & 6202.99 & 331.61 & 326.67 \\
\hline $\begin{array}{c}\text { Sound pressures } \\
\text { after installation, } \mathrm{Pa}\end{array}$ & 6215.83 & 330.57 & 325.82 \\
\hline
\end{tabular}

reasonable, and that the parameters of the transducer meet the tool requirements.

The debugging system can effectively test the sensitivities and directivities of every piezoelectric vibrator in the receiving mandrel. The experimental results of sensitivity are shown in Table 2. Here, we have listed the results of four piezoelectric vibrators only from the maximum spacing receiver $\mathrm{R} 8$, since the results of receiver R1-R7 are similar to the receiver R8. Overall, the sensitivity of each piezoelectric vibrator before and after installation is consistent, implying that the structure and assembly technologies of the receiving mandrel do not affect the characteristics of the piezoelectric vibrators.
Table 2 Comparison of the sensitivity of the piezoelectric vibrators in the maximum spacing receiver $\mathrm{R} 8$ in one receiving mandrel

\begin{tabular}{ccccc}
\hline & $\mathrm{R} 8.1$ & $\mathrm{R} 8.2$ & $\mathrm{R} 8.3$ & $\mathrm{R} 8.4$ \\
\hline $\begin{array}{c}\text { Sensitivity before installation, } \\
\mu \mathrm{V} / \mathrm{Pa}\end{array}$ & 70.73 & 70.32 & 70.95 & 70.65 \\
\hline $\begin{array}{c}\text { Sensitivity after installation, } \\
\mu \mathrm{V} / \mathrm{Pa}\end{array}$ & 70.61 & 70.29 & 70.85 & 70.47 \\
\hline
\end{tabular}

In the design and production of the tool, analog channel consistency is a very important factor. Hence the key parameters and parts are strictly tested and selected during the tool production. The debugging system proposed in this study can evaluate the consistency of eight analog channels. The phase sensitive detection extracts the amplitude and phase from the sampling data, thereby establishing consistency in the evaluation of the analog channels. Table 3 shows the experimental results of consistencies in the eight channels of one tool. According to the experimental results, the parameters of the circuit should be fine-tuned to get the better consistency for each channel.

Table 3 Experimental results of consistencies in the eight channels of one tool

\begin{tabular}{ccccccccc}
\hline & Channel 1 & Channel 2 & Channel 3 & Channel 4 & Channel 5 & Channel 6 & Channel 7 & Channel 8 \\
\hline $\begin{array}{c}\text { Amplitude } \\
\text { mV }\end{array}$ & 2798.52 & 2798.96 & 2798.31 & 2798.05 & 2799.32 & 2799.65 \\
\hline $\begin{array}{c}\text { Phase } \\
\text { Deg }\end{array}$ & 176.3 & 176.5 & 176.6 & 176.3 & 176.8 & 176.2 \\
\hline
\end{tabular}

\section{Conclusions}

The debugging system proposed in this study can provide specialized testing and analysis at the system-level, functional section level, and local circuit unit level, thereby ensuring high quality and efficiency in the manufacturing process, maintenance, and field use of MPAL.

Compared with conventional bus card interfaces, such as UART, USB, and so on, the ARM-based network connection overcomes the shortcomings of interconnection distance, data bandwidth, and reliability.

Test results indicate that the data transmission rate of the system is greater than $1 \mathrm{MB} / \mathrm{s}$, thereby completely meeting the requirements of the current data transmission rate between the tool system and the wireline telemetry device.

The efficacy of the proposed debugging system has been verified by applying it for the production, maintenance, and field operations of MPAL. The debugging system satisfies the test requirements of various levels and shows good performance.

If the system control software is upgraded to suit the corresponding tool, then the debugging system can also be used to test other logging tools with similar interfaces, such as the azimuthally acoustic bond tool. Although the working principle of other multi-pole array acoustic logging tools (for example XMAC II) is similar, the hardware interface and communication protocol of the tools are significantly different. So, it is highly challenging to debug the tools with a variety of interfaces in a test platform.

\section{Acknowledgements}

This study is supported by National Science Foundation of China (61102102, 11134011, 11204380 and 11374371), Major National Science and Technology Projects (2011ZX05020002), PetroChina Innovation Foundation (2014D-5006-0307), Science and Technology Project of CNPC (2014A-3912 and 2011B-4001) and the Foundation of China University of Petroleum (KYJJ2012-05-07). The authors also would like to thank three anonymous reviewers for their helpful discussion and suggestions.

\section{References}

Altera Corporation. MAX II device handbook. [2009-08]. http://www. altera.com.cn/literature/hb/max2/max2_mii5v1.pdf

Che X H, Zhang H L, Qiao W X, et al. Numerical study on scanning radiation acoustic field in formations generated from a borehole. Science in China (Ser. G). 2005. 48(2): 247-256 (in Chinese)

Chen W J, Wang J and Nie Z P. Study on the calibration of receiving channels and the beamforming technology for wideband digital array antennas. Acta Electronica Sinica. 2013. 41(3): 582-586 (in Chinese)

Chen X L and Tang X M. Numerical study on the characteristics of acoustic logging response in the fluid-filled borehole embedded in 
crack-porous medium. Chinese Journal of Geophysics. 2012. 55(6): 2129-2140 (in Chinese)

Gong S L, Roh H T and Lee J W. Cross-layer and End-to-End optimization for the integrated wireless and wireline network. Journal of Communications and Networks. 2012. 14(5): 554-565

Jin S, Han K and Choi S. Idle mode for deep power save in IEEE 802.11 WLANs. Journal of Communications and Networks. 2010. 12(5): 480-491

Ju X D and Li H Y. Design and application of a logging panel based on embedded technology. Journal of the University of Petroleum, China. 2005. 29(2): 30-41 (in Chinese)

Ju X D, Cheng X Y, Lu J Q, et al. Design of test-bench system for logging tools based on embedded structures. Well Logging Technology. 2009. 33(3): 270-275 (in Chinese)

Ju X D, Qiao W X, Cheng X Y, et al. Embedded data acquisition and control system design based on Ethernet connection. Computer Measurement \& Control. 2010. 18(5): 1073-1078 (in Chinese)

Ju X D, Qiao W X, Li Y X, et al. Electronic system design of a multipole acoustic logging tool. Well Logging Technology. 2008. 32(1): 61-64 (in Chinese)

Lattice Semiconductor Corp. ispLSI and pLSI 1016E Data Sheet. [200608-25]. http://www.latticesemi.com/lit/docs/datasheets/cpld/1016e. pdf

Liu C, Qin X W, Zhang S H, et al. Proportional-fair downlink resource allocation in OFDMA-based relay networks. Journal of Communications and Networks. 2011. 13(6): 633-638

Liu H B, Lin N, Pan S S, et al. High sensitivity, miniature, full 2-D anemometer based on MEMS hot-film sensors. IEEE Sensors Journal. 2013. 13(5): 1914-1920

Lu J Q. Electronic system research and designing of multi-pole array acoustic logging tool. Beijing: Ph.D. Dissertation. China University of Petroleum. 2007 (in Chinese)

Lu J Q, Ju X D and Cheng X Y. Design of a cross-dipole array acoustic logging tool. Petroleum Science. 2008. 5(2): 105-109

Lu J Q, Ju X D, Men B Y, et al. Study on test method for transmitter mandrel of acoustic logging tool based on ARM. Well Logging Technology. 2010. 34(4): 389-392 (in Chinese)

Lu J Q, Ju X D and Men B Y. Design of test platform for receiver mandrel of multi-pole array acoustic logging tool. 2012 9th International Conference on Fuzzy Systems and Knowledge Discovery (FSKD2012). 2012. 2673-2676

Matthew N and Stones R. Beginning Linux Programming, 4th Edition. Indiana: Wiley Publishing, Inc. 2007

Men B Y, Ju X D, Qiao W X, et al. Design of high temperature test system for high-accurate ADC based on embedded structures. Journal of China University of Petroleum (Edition of Natural Science). 2011. 35(3): 67-71 (in Chinese)

Peng S M, Su Y K, Ji L W, et al. Photoelectrical and noise characteristics of $\mathrm{ZnO}$ nanowire networks photosensor. IEEE Sensors Journal. 2011. 11(5): 1173-1177

Qiao W X, Ju X D, Che X H, et al. Multipole acoustic logging transmitter structurized by annular array. Journal of the University of Petroleum, China. 2006. 30(5): 33-41 (in Chinese)

Qiao W X, Ju X D, Che X H, et al. Progress in acoustic well logging technology. Well Logging Technology. 2011. 35(1): 14-19 (in Chinese)

Samsung Electronics. S3C44B0X RISC MICROPROCESSOR ERRTA. [2001-12-19]. http://www.datasheetcatalog.org/datasheet/ SamsungElectronic/mXwsqyy.pdf

Su Y D, Tang X M, Hei C, et al. An equivalent-tool theory for acoustic logging and applications. Applied Geophysics. 2011. 8(1): 69-78

Tang X M and Cheng C H. Quantitative borehole acoustic methods. Amsterdam: Elsevier. 2004. 1-10

Tang X M and Patterson D J. Single-well S-wave imaging using multicomponent dipole acoustic-log data. Geophysics. 2009. 74(6): WCA211-WCA223

Tang X M and Wei Z T. Single-well acoustic reflection imaging using far-field radiation characteristics of a borehole dipole source. Chinese Journal of Geophysics. 2012. 55(8): 2798-2807 (in Chinese)

Tang X M, Zheng Y and Patterson D. Processing array acoustic-logging data to image near-borehole geologic structures. Geophysics. 2007. 72(2): E87-E97

Wang B. An invariant region-shape descriptor based on Fourier transform. Acta Electronica Sinica. 2012. 40(1): 84-88 (in Chinese)

Wang R J, Qiao W X and Ju X D. Numerical study of formation anisotropy evaluation using cross dipole acoustic LWD. Chinese Journal of Geophysics. 2012. 55(11): 3870-3882 (in Chinese)

Wang R J, Qiao W X, Ju X D, et al. Experimental study of the acoustic field in the borehole surrounded by HTI formations excited by dipole sources with different orientations. Chinese Journal of Geophysics. 2013. 56(2): 707-717 (in Chinese)

Wu J P, Qiao W X, Che X H, et al. Experimental investigation on temperature performance consistency of piezoelectric benders used in acoustic well logging. Well Logging Technology. 2012. 36(2): 109-113 (in Chinese)

Wu J P, Qiao W X, Che X H, et al. Experimental study on the radiation characteristics of downhole acoustic phased combined arc array transmitter. Geophysics. 2013. 78(1): D1-D9

Yang W D and Cai Y M. On the performance of the block-based selective OFDM decode-and-forward relaying scheme for 4G mobile communication systems. Journal of Communications and Networks. 2011. 13(1): 56-62

Ye P, Zeng H, Tian S L, et al. Research on the real time calibration technology for high-speed. Acta Electronica Sinica. 2013. 41(1): 166170 (in Chinese)

(Edited by Hao Jie) 